\title{
Ständige Impflkommission
}

\section{Neuer Impfkalender: Das hat sich geändert}



\) Dieses Jahr wird der Kalender des Robert Koch-Institut (RKI) zwar nicht ausgeweitet, aber bei einigen wichtigen Impfungen wurden Empfehlungen angepasst (Epi Bull 2017; 34: 333).

Hepatitis-A und B: Impfschutz gegen Virushepatitis empfiehlt die Ständige Impfkommission (STIKO) nun auch Ehrenamtlichen und nicht nur Berufstätigen mit Expositionsrisiko. Ausdrücklich genannt werden Auszubildende, Studierende und Praktikanten. Hier- mit wird klargestellt, dass die Indikation zur Impfung anhand des Expositionsrisikos bei einer Tätigkeit zu beurteilen ist und nicht auf bestimmte Berufsgruppen beschränkt ist.

Influenza: Die Präferenz für den Lebendimpfstoff als Nasenspray bei Kindern von zwei bis sechs Jahren ist endgültig zurückgezogen worden. In den letzten Jahren war im Vergleich zu den inaktivierten Standard-Impfstoffen keine überlegene Wirksamkeit mehr nachweisbar.

Tetanus: Eine Auffrischimpfung bei geringfügigen und sauberen Wunden wird von der STIKO nur noch dann empfohlen, wenn seit der letzten Impfung mehr als zehn Jahre vergangen sind. Damit wurde die 2016 erfolgte Absenkung der Frist von zehn auf fünf Jahre rückgängig gemacht.

Herpes zoster: Seit 2013 ist in Deutschland ein Lebendimpfstoff gegen Herpes zoster (HZ) und postherpetische Neural- gie für Menschen im Alter ab 50 Jahren verfügbar. Die STIKO lehnt zurzeit eine Empfehlung für diese Vakzine als Standardimpfung ab, weil die Daten zu Wirksamkeit, Schutzdauer und Sicherheit des Impfstoffs das Gremium nicht überzeugt haben. So nimmt die Wahrscheinlichkeit an HZ zu erkranken und die Schwere der Erkrankung mit dem Alter deutlich zu, hingegen nimmt die Wirksamkeit der Impfung mit dem Alter ab. Zudem ist die Schutzdauer nur für wenige Jahre belegt.

Neu eingefügt hat die STIKO einen Abschnitt zu Impfungen von Patienten mit Immundefizienz und Immunsuppression. Für betroffene Patienten erarbeitet das Gremium momentan Anwendungshinweise für Impfungen. Diese sollen bis Anfang 2018 veröffentlicht werden.

(wg)

www.rki.de/epidbull

\section{Interkulturelles Pflegemanagement}

\section{Mit Master-Kompetenz Mitarbeiter und Patienten fördern}

") In einer vernetzten Welt ist auch das Gesundheitssystem mit Menschen aus den unterschiedlichsten Kulturkreisen konfrontiert - sowohl als Mitarbeiter als auch als Patienten und deren Angehörige. Mit einem Masterlehrgang qualifiziert die Akademie für Weiterbildung der FH Oberösterreich die Teilnehmer zum Interkulturellen Pflegemanagement (IKM). Denn Führungs- personen in der Pflege benötigen zunehmend Kompetenzen, um Konzepte zu schaffen, die der kulturellen Herkunft von Mitarbeitern als auch von Klienten und Patienten Rechnung tragen. Interkulturelle Kompetenzentwicklung und eine kultursensible Pflege setzt interkulturelles Management voraus. Der fünfsemestrige Masterlehrgang qualifiziert Pflegende des ge-

\section{Die Führung ist weiblich}

\section{APOLLON Hochschule bestellt erstmals Präsidentin}

» Mit Prof. Dr. Johanne Pundt hat die Apollon Hochschule zum 1. September 2017 eine kompetente, erfahrene und breit vernetzte Präsidentin an die Spitze der privaten Fernhochschule berufen. Sie löst den Gründungspräsidenten Prof. Dr. Bernd Kümmel im Rahmen einer Altersnachfolge ab. Die Sozial- und Gesundheitswissenschaftlerin Pundt führt die Hochschule für sechs Jahre zusammen mit den beiden Geschäftsführern der Trägergesellschaft. Sie ist für die Steuerung und Entwicklung der Forschung und Lehre der APOLLON Hochschule verantwortlich, vertritt die Hochschulbelange nach innen sowie außen und leitet als Vorsitzende den Senat und das Kuratorium. Neben ihrer hobenen Dienstes für Gesundheitsund Krankenpflege zur Ausübung einer Pflegedienstleitung nach $\S 72$ GuKG mit der Schwerpunktsetzung Interkulturalität. Der nächste Lehrgang in Linz beginnt im Januar 2018 und dauert fünf Semester. Anmeldeschluss ist am 15. November 2017.

\section{http://bit.ly/2d9gzWP}

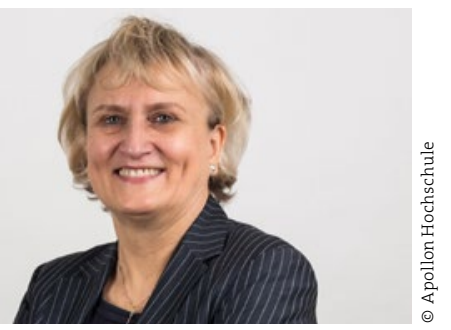

neuen Funktion steht sie der Hochschule weiter als Dekanin und Studiengangsleiterin zur Verfügung.

www.apollon-hochschule.de 